\title{
Tre onde
}

\section{Three waves}

Giovanni Lodi

Dipartimento di Scienze Biomediche, Chirurgiche e Odontoiatriche

Università degli Studi di Milano

via Beldiletto 1/3 Milano 20142

Italia

giovanni.lodi@unimi.it

tel. + 390250319021

fax. +390250319041

Esattamente due anni fa, nell'ottobre 2017, il governo degli Stati Uniti dichiarava "emergenza sanitaria nazionale" un'epidemia che dal 1999 ad oggi ha provocato 400 mila morti e che continua ad uccidere con un ritmo di 130 decessi al giorno.

Sono i morti da overdose da oppioidi, vittime di una storia complicata in cui sostanze capaci di dare dipendenza in maniera rapida e inesorabile hanno contribuito in modo significativo al declino dell'aspettativa di vita che per tre anni di seguito si è verificato tra i cittadini statunitensi, evento unico tra i paesi della parte più ricca del mondo. Un bambino nato negli Stati Uniti nel 2017 vivrà fino a 78,6 anni, in Italia supererà facilmente gli 82.

La storia è complicata perché l'epidemia è il risultato di quelle che i Centers for Disease Control descrivono come tre onde distinte di morti da overdose, cominciate nel 1990 con quella da farmaci antidolorifici, a cui si è aggiunta quella da eroina degli anni '10 e infine quella da oppioidi sintetici prodotti e venduti illegalmente iniziata nel 2013. Questi tre gruppi di sostanze hanno causato solo nel 2017 più di 70 mila morti, con un incremento del $10 \%$ rispetto all'anno precedente. Una strage continua che sembra non sembra dare segni di declino.

Una grossa responsabilità di tutte queste morti è quindi da ascrivere alle prescrizioni dei medici statunitensi, come dimostrano i numeri delle ricette per uno dei farmaci maggiormente implicati, l'OxyContin, passate da 670.000 a 6,2 milioni all'anno, nel periodo compreso tra il 1997 e il 2002. Purtroppo di questo abuso e delle sue tragiche conseguenze, sono state riconosciute responsabili aziende farmaceutiche che con metodi leciti e non, hanno promosso queste sostanze, enfatizzandone i benefici, ma soprattutto minimizzandone i rischi.

L'American Dental Association ha riconosciuto il contributo dei nostri colleghi statunitensi alla crisi.

Tradizionalmente grandi prescrittori di oppioidi per il controllo del dolore dentale, i dentisti US hanno deciso si autoregolamentarsi stabilendo linee di comportamento in grado di limitare le prescrizioni e ridurre il rischio di dipendenza.

In Italia fortunatamente nulla di tutto questo è accaduto, e speriamo non accada mai. Questo però non significa che qualcuno non ci abbia provato, come sembrerebbero suggerire alcune cronache giudiziarie. Se siete curiosi, cercate in rete "pain league".

Buona lettura, 